\title{
Plasma Emission Codes: Comparisons and Critiques
}

\author{
HELEN E. MASON \\ Department of Applied Mathematics and Theoretical Physics, Silver Street, Cambridge \\ CB3 9EW, UK
}

A great deal of effort in recent years has gone into the development of spectroscopic techniques to probe the physical parameters of solar, stellar and other astrophysical plasmas. One aspect of this work is the calculation of plasma emission codes which are used to study EUV spectral lines. These codes require the input of a large amount of atomic data. In this paper, we present an overview of the atomic processes involved and an assessment of the accuracy of the parameters which are incorporated into different emission codes.

\section{Introduction}

The task of this review has been made significantly easier because a workshop on Plasma Emission Codes was held in Napa Valley on November 2nd-5th, 1994. The proceedings are being published as a report (Brickhouse et al. 1995). A benchmark comparison was made between different plasma emission codes for wavelength ranges relevant to recent astrophysical observations. In this review, we give an overview of the strengths and weaknesses of the various plasma emission codes; we discuss the problem areas identified at the Napa Valley meeting, in particular wavelength ranges where codes produce different synthetic spectra or where there are substantial discrepancies with observations; we give a user's guide to the relevant atomic data; we ask what has been learnt from the past twenty years of solar observations and finally we look to future developments in the plasma codes for solar spectral and astrophysical observations. An extensive review of spectroscopic diagnostics in the UV and EUV wavelength ranges for solar and stellar plasmas has recently been published by Mason \& Monsignori Fossi (1994).

\section{Napa Valley Workshop}

The Napa Valley workshop made benchmark comparisons for the wavelength ranges $0.1-10 \mathrm{KeV}(A S C A) ; 40-85 \AA(D X S) ; 85-125 \AA$ and $125-180 \AA$ (EUVE); in parenthesis are the relevant astrophysical observations. The plasma emission codes which were included in the comparative study were MEKA (Mewe, Kaastra et al. ); KM (Kato \& Masai); BRS (Brickhouse, Raymond \& Smith); MFL (Monsignori Fossi \& Landini). These codes have similar features - they all deal with the continuum and line emission from hot, optically thin plasmas. The formulations used for continuum emission (free-free, free-bound, two-photon) are in general agreement, so in this review we shall concentrate on the approximations used to compute the line emission spectrum. The main features to be considered are: the treatment of ionisation and recombination processes; the type of model ion used for the level populations (treatment of metastable levels and highly excited levels); the representation of electron collisional excitation rates; inclusion of proton rates; the contribution of di-electronic and inner shell satellite lines. The main features of each code are described below. 


\subsection{MEKA, XSPEC, SPEX}

The first version of the MEKA code, published by Mewe (1972), covered the wavelength range 1-300 $\AA$. Updated versions, including improved atomic data were published by Mewe \& Gronenschild (1981), Mewe et al. (1985). The electron density dependence of the population for the lower levels are allowed for together with a correction for cascades from higher levels.

The electron excitation rates are represented by an approximation based on the $\bar{g}$ formula (Van Regemorter 1962). This is used by Mewe and other authors to estimate the intensity for the solar lines, so we discuss it here in some detail.

The averaged collision strength, $\Upsilon$, is defined as

$$
\Upsilon_{i j}=\int_{0}^{\infty} \Omega_{i j} \exp \left(\frac{-E_{j}}{k T}\right) d\left(E_{j} / k T\right)
$$

where $\Omega_{i j}$ is the collision strength for the transition $i$ to $j, E_{j}$ is the electron energy relative to the final state $j, k$ is the Boltzmann constant and $T$ is the electron temperature of the plasma.

The electron collision rate is then given by the well known formula

$$
q_{i j}=8.6310^{6} T^{-0.5} \exp \left(-E_{i j} / k T\right) \Upsilon_{i j} / \omega_{i}
$$

The averaged collision strength $\Upsilon$ (which Mewe calls $\bar{\Omega}$ ) is related to $\bar{g}$ by

$$
\Upsilon_{i j}=\frac{8 \pi}{\sqrt{ } 3} \frac{\omega_{i} f_{i j}}{E_{i j}} \bar{g}
$$

where $\omega_{i} f_{i j}$ is the radiative oscillator strength and $E_{i j}$ is the energy difference between levels $i$ and $j$.

It should be emphasised that the $\bar{g}$ formulation was developed for dipole transitions. Mewe (1972) extended its use to forbidden transitions. He uses an expression of the form

$$
\bar{g}=A+\left(B y-C y^{2}+D y^{3}+E\right) e^{y} E_{1}(y)+(C+D) y-D y^{2}
$$

for both allowed and forbidden transitions. The parameters A, B, C, D, and $\mathbf{E}$ are derived by fitting to available electron excitation rates. The parameter $y$ is $E_{i j} / k T$, and $E_{1}(y)$ is a standard exponential integral. A serious problem arises when electron excitation rates are not available and the parameters must be guessed.

Doschek \& Cowan (1984) produced a synthetic spectrum from a composite of observed solar spectra. Mewe et al. (1985) re-normalised their semi-empirical parameters to fit this spectrum better.

The synthetic spectra developed by Mewe and coworkers include di-electronic recombination and inner shell satellite lines. The plasma emission code uses the most recent ionisation and recombination rates and is able to deal with time-dependent processes. Kaastra \& Mewe (1993) reported on an updated version of these synthetic spectra called XSPEC. In 1996, a revised and extended version of these codes, called SPEX will be available. This will cover the wavelength range 1-2000 $\AA$, with improved atomic data including new $n=2$ to $n=3, n=4$ electron excitation data.

\section{2. $K M$}

Kato(1976) published a synthetic spectrum covering the wavelength range 1-250 $\AA$, this was later updated and extended by Masai $(1984 \mathrm{a}, 1984 \mathrm{~b})$. The treatment was similar to that of Mewe and coworkers, using the $\vec{g}$ formulation for electron excitation and some 
allowance for cascade effects. Again time dependent ionisation, using standard ionisation and recombination rates was incorporated. The most recent update is by Masai (1994), using the Arnaud \& Raymond (1992) ionisation and recombination calculations for the iron ions.

\section{3. $B R S$}

The Raymond \& Smith $(1977,1988)$ synthetic spectra covered the wavelength range 1$200 \AA$. The $\bar{g}$ formulation similar to Mewe's was used for the electron collisional excitation and the metastable levels were also treated in a similar way to Mewe. The formulation for ionisation (+excitation/auto-ionisation) was based on the Exchange Classical Impact Parameter method and the re-combination processes were calculated with a refined form of the Burgess General Formula. The codes can deal with time dependent processes. Recently Brickhouse et al. (1995) have published new synthetic spectra for the iron ions in the wavelength range 70-600 $\AA$ using the Arnaud \& Raymond (1992) ionisation balance and the best available electron excitation rates. The statistical equilibrium equations are solved for each ion. Future improvements to this work include a study of other elements and an extension of the wavelength range to cover the $\mathrm{X}$-ray wavelength region.

\section{4. $M F L$}

Landini \& Monsignori Fossi (1972, 1990, 1991) published a synthetic spectrum in the wavelength range $1-2000 \AA$. These authors used a $\vec{g}$ formulation similar to Mewe and coworkers. Their ionistaion and recombination rates were a little different. Recent work by Monsignori Fossi \& Landini (1994) uses the ionisation rates of Arnaud \& Raymond (1992) for the iron ions. They solve the statistical equilibrium equations for the low lying levels of each ion and represent the electron excitation rates using the Burgess \& Tully (1992) method, which is described in a later section.

\subsection{Problems Identified at the Napa Valley Workshop}

In the $\mathrm{X}$-ray wavelength range, large discrepancies between the spectral codes were found to be due primarily to the use of different formulations for the ionisation balance. In addition, a major problem was identified for the intensity ratios of the transitions between $n=2$ to $n=3$ levels and $n=2$ to $n=4$ levels of highly ionised systems. In the $40-85 \AA$ range, there were discrepancies between the intensities for the Fe XV and Fe XVI spectral lines. In the 85-125 $\AA$ region some discrepancies were identified for the iron ions Fe XVIIIXXII and it was noted that the Ni ions need more theoretical work. The wavelength range $>200 \AA$ was not investigated.

\section{Accuracy of Atomic Calculations}

The atomic data required for the analysis of astrophysical spectra includes: energy levels and radiative data; electron and proton excitation rates; ionisation and re-combination rates. Recent progress in these areas includes laboratory measurements of atomic parameters, for which only theoretical calculations previously existed. Although in several cases the independent theoretical calculations had converged to what was assumed to be a high accuracy result, unfortunately the laboratory measurements give a different value. It is beyond the scope of this review to discuss the details, but simply to sound a note of caution.

\subsection{Solar Context}

In the 1970 s, many new atomic physics calculations were prompted by the Skylab UV observations and the HRTS (High Resolution Telescope Spectrometer) rocket instru- 
ment. The emphasis in the 1980 s was on solar flare spectra, stimulated by the success of projects such as the Solar Maximum Mission, SOLEX, SOLFLEX and HINOTORI. These instruments obtained high resolution spectra in the $\mathrm{X}$-ray wavelength regions, below $25 \AA$ as well as the UV (1000-2000 $\AA$ ). The instruments on the YOHKOH satellite have recently provided some fascinating X-ray images and spectra. Interest in the UV wavelength range has also been revived with the successful flights of the SERTS (Solar EUV Rocket Telescope and Spectrometer) (Neupert et al. 1992; Thomas \& Neupert 1994). Much effort has been directed towards a major satellite mission, the Solar Heliospheric Observatory ( $S O H O$ ) to be launched in October 1995. This will carry several UV spectroscopic instruments $(160-1600 \AA)$, including the Coronal Diagnostic Spectrometer (CDS) (Harrison \& Sawyer 1992) and the Solar Ultraviolet Measurement of Emitted Radiation (SUMER) instrument (Lemaire et al. 1992).

\subsection{Ionisation Balance}

The large discrepancies for the iron ions between Arnaud \& Raymond (1992) and Arnaud $\&$ Rothenflug (1985) require further investigation. Shifts on the order of $0.1-0.2 \mathrm{dex}$ are found in the temperatures corresponding to peak ion abundances. The peak values of the ionisation ratio typically differ by $10-20 \%$. Detailed studies of the formulations used ionisation and recombination rates can be found in Raymond (1988), Mewe (1990), and the recent paper by Jordan (1995). The main questions to be addressed are the more accurate account of indirect processes in the ionisation rates (Moores \& Reed 1995) and the treatment of electron density dependence and alternative decay channels in the di-electronic recombination rates.

\subsection{Electron Excitation Rates}

Accurate electron scattering calculations are now available for many of the ions which are abundant in the solar corona and transition region. These were reviewed at an atomic data assessment workshop held in Abingdon, UK, in 1992 (Lang 1994).

\subsubsection{Fe $X V$ and Fe $X V I$}

Below we consider the accuracy of the representations of these atomic data in the various plasma emission codes, in particular for Fe XV and Fe XVI which were reviewed by Badnell \& Moores (1994).

Sampson \& Zhang (1992) studied the use of the Van Regemorter formula for collision strengths. Their abstract states, "It is found to be a very poor approximation, especially for $\Delta n \geq 1$ excitation transitions from levels $l<n-1$, and the recommendation is made that with recent advances in calculation procedures and available accurate atomic data, use of the Van Regemorter formula be discontinued." This approximation is only applicable to optically allowed (electric dipole) transitions. There is no justification for applying this formulation to other types of transitions.

The ion Fe XVI is abundant in solar active regions and flares. Strong spectral lines from this ion have been observed over a wide wavelength range. The transitions $3 \mathrm{~s}-$ $3 \mathrm{p}$ and $3 \mathrm{p}-3 \mathrm{~d}$ fall between 250 and $365 \AA$ and lines from transitions between $\mathrm{n}=3$ to $\mathrm{n}=4, \mathrm{n}=3$ to $\mathrm{n}=5$ fall between 30 and $80 \AA$. These lines have also been observed in stellar spectra. Very few solar spectra have been recorded in this wavelength region, however, spectra in the 10-100 $\AA$ wavelength region have been obtained for a solar flare with the XSST rocket instrument (Acton et al. 1985). Cornille et al. $(1993,1995)$ have compared the theoretical and observed intensity ratios for the strong Fe XVI lines which were prominent in these spectra. They note that the spectral lines corresponding to transitions between $3 \mathrm{p}-4 \mathrm{~s}(62.88 \AA, 63.71 \AA), 3 \mathrm{p}-4 \mathrm{~d}(54.13 \AA, 54.72 \AA, 54.77 \AA)$ and $3 \mathrm{~d}-$ 
$4 \mathrm{f}(66.25 \AA, 66.36 \AA)$ are as strong as the spectral lines corresponding to $3 \mathrm{~s}-4 \mathrm{p}(50.35 \AA$, $50.56 \AA$ ) transitions. There is also an important temperature sensitivity for the spectral line intensity ratios. A similar phenomena is seen for the spectral lines for $n=3$ to $n=5$ transitions. Cornille et al. (1995) give a comparison of their theoretical intensity ratios and those obtained using the $\bar{g}$ formulation given in the various plasma emission codes. The closest agreement is found for Doschek \& Cowan (1985), which is to be expected since their values are obtained from the solar observations! The synthetic spectra of Mewe et al. (1985) and Landini \& Monsignori Fossi (1989) give major discrepancies (up to a factor 10) for the intensity ratios of the Fe XVI lines relative to the transition $3 \mathrm{~s}-4 \mathrm{p}$. The $\bar{g}$ formulation used in these plasma emission codes gives a very poor representation of the Fe XVI spectral lines between 50-60 $\AA$. Very recent versions of these codes have better representations for the Fe XVI excitation rates, but what about other ions, such as Fe XV, for which no accurate atomic data has yet been published?

Electron scattering calculations for $\mathrm{Fe} X \mathrm{X}$ have recently been completed by Bhatia et al. (1995) including the $n=3$ to $n=4$ transitions. They find that the electron collision rates for the monopole and quadrupole transitions are large in comparison to the values for the dipole transition $3 s^{2}{ }^{1} S_{0}-3 s 4 p{ }^{1} P_{1}(52.91 \AA)$. They calculate intensity ratios for the Fe XV spectral lines and compare these with the values obtained in the solar flare spectrum from XSST. The agreement between the theoretical and observed values is good. In fact, it is possible to identify the very strong line at $69.65 \AA$ as the Fe XV transition $3 s 4 s^{1} S_{0}-3 s 3 p{ }^{1} P_{1}$. This is almost a factor of 10 stronger than the resonance line at $52.91 \AA$. The theoretical and observed intensity ratios for these lines are completely different from those estimated by the synthetic spectrum codes, even the most recent ones!

Since the Fe XV and Fe XVI X-ray lines have not been extensively studied with solar spectra, it is perhaps not so surprising that such major inconsistencies have gone unnoticed until very recently, however the plasma emission codes have been used to analyse astrophysical data, for example EXOSAT observations of stellar coronae (cf., Schrijver et al. 1989; Lemen et al. 1989); ROSAT (Schmitt 1992) and DXS Bragg Crystal Spectrometer (44-83 $\AA$; Sanders 1995). For such analyses, large inaccuracies in the simulated spectra for Fe XVI and Fe XV could lead to a misinterpretation of the distribution of material as a function of temperature.

\subsubsection{Fe $X V I I I-F e X X I V$}

Extensive electron scattering calculations have been carried out for the iron ions Fe XVIII-XXIII, both within the $n=2$ configurations and between the $n=2$ to $n=3$ configurations. Solar observations in the $90-150 \AA$ wavelength range are sparse, but a very good flare spectrum was obtained with the with OSO-5. The diagnostic potential of these iron lines is discussed in Mason et al. (1984), together with references to atomic calculations. Electron excitation rates have been calculated for iron and other elements (e.g., $\mathrm{Ne}, \mathrm{Mg}, \mathrm{Si}, \mathrm{S}, \mathrm{Ca}$ ) of solar interest by Mason, Bhatia, Doschek, Dubau and colleagues using the University College London distorted wave program. Many calculations have also been carried out with the more sophisticated RMATRX, close coupling program, developed at Queens' University Belfast. The atomic data for the $2 s^{2} 2 p^{n}--2 s 2 p^{n+1}$ transitions is very accurate and references can be found in the reviews in Lang (1994).

The recent versions of the plasma emission codes are all based on these atomic data. There is no obvious reason for discrepancies between the synthetic spectra. One important process which is not accounted for in these codes is that of proton excitation. It is particularly important for these highly ionised systems and should be included in the statistical equilibrium equations. 
The $n=2$ to $n=3$ transitions in Fe XVIII-Fe XXIV give rise to spectral lines in the $\mathrm{X}$-ray wavelength range $(10-20 \AA)$. These were recorded by a different instrument on OSO-5. These electron scattering calculations are more complex than those within the $\mathrm{n}=2$ configurations, but the accuracy of available atomic data should be good. For several ions electron excitation rates have also been calculated for the $n=2$ to $n=4$ transitions.

\subsubsection{Fe $I X-F e X I V$}

The ions Fe IX-Fe XIV give rise to a whole host of spectral lines in the UV wavelength range, of particular interest to the SOHO-CDS instrument. The available atomic data for these ions was reviewed by Mason (1994). These recommended data were used by BRS, MFL. It was pointed out by Mason that some of the available calculations had very limited accuracy. Since that review, new atomic data have been published for several of these ions. For example, the new calculations for $\mathrm{Fe} \mathrm{X}$ are reviewed by Young et al. (1995) and a comparison is made between theoretical intensity ratios and solar (SERTS) and stellar (EUVE) spectra.

An international collaboration called the Iron Project (co-ordinated by D. Hummer) aims to calculate accurate electron excitation data for these and many other ions using the most accurate methods available. A series of papers reporting on this work is being published in Astronomy and Astrophysics. As part of this project, Storey et al. (1995) report on new calculations for the famous Fe XIV green coronal line (5303 $\AA$ ). They use a very accurate target and include all the important resonance contributions to the collision strength.

\subsection{Atomic Data Assessment Program}

Burgess \& Tully (1992) have developed a graphical procedure to fit the collision strengths and to obtain upsilon ( $\Upsilon$ ) (OMEUPS). The collision strengths (or Upsilon's) are plotted on a reduced energy (or temperature) scale such that 0 corresponds to threshold (or zero temperature)and 1 corresponds to infinite energy (or temperature). The correct functional behaviour of the collision strength (or upsilon) on energy (or temperature) which depends on the type of transition (i.e., dipole, exchange, forbidden) is taken into account. The high energy (or temperature) limit is obtained from the Bethe or Born approximations. A five point spline is fitted to the reduced data by a least squares procedure. The advantage and beauty of this method is its precision and simplicity. Any errors or problems with the atomic data are immediately obvious. Published data from different authors are all stored in the same format, with only five points required to reproduce the whole energy (or temperature) range. This method is being used by several groups to provide an atomic databank which will be made available to the solar and astrophysics community.

\section{Future Directions}

\subsection{Synthetic Spectrum Project}

A new synthetic spectral code $(45-2000 \AA)$ is being prepared as part of a collaboration between Dere, Monsignori Fossi, Mason and coworkers using the graphical assessment method of Burgess \& Tully (1992). Dere has written a version of the Burgess \& Tully assessment method which runs in IDL (Interactive Data Language) which is extensively used for solar analyses. The immediate aim is to prepare for the analyses of spectra from SOHO. It is anticipated that the essential data and basic programs should be ready by the end of 1995. Significant progress has been achieved through the willingness of atomic physicists to make their results available in digital form. 


\subsection{CDS and SUMER Scientific Software}

Preparations for $S O H O$ have been in progress for several years. The aim is to build a package of analysis software for spectroscopic diagnostics. An important component of this project is the Atomic Data and Analysis Structure (ADAS) which was developed by Summers for fusion research and is now being adapted for solar application. ADAS is a very powerful suite of subroutines which solves the collisional radiative model for equilibrium or non-equilibrium conditions. It contains a comprehensive atomic data analysis, storage and processing procedure. All the recommended atomic data from Lang (1994) and more recent calculations (eg Iron Project) will be incorporated together with atomic data assessed using OMEUPS. ADAS is capable of producing ionisation balance calculations together with low lying level populations. These are being incorporated into solar analysis programs for spectroscopic diagnostics (electron temperature and density determination, emission measure analyses, elemental abundance determinations).

\section{Conclusion}

Maybe at the end of this review one might expect a league table for the different synthetic spectral codes. This would result in the loss of some good relations and collaborators! The task of assembling the synthetic spectral codes is formidable. Each project has its own strengths and weaknesses. The main conclusion of this review is rather obvious, that the BEST plasma emission are the ones which are able to represent the atomic rates the most accurately. The question still arises with regard to missing atomic data. Fortunately, the atomic physics community is always receptive to suggestions for interesting and useful new computations.

The financial support of PPARC is acknowledged.

\section{REFERENCES}

Acton, L. W., Bruner, M. E., Brown, W. A., Fawcett, B. C., Schweizer, W. \& Speer, R. J. 1985, ApJ, 291, 865

ARnaUd, M. \& Raymond, J. C. 1992, ApJ, 398, 39

ARNaUd, M. \& Rothenflug, R. 1985, A\&AS, 60, 425

Badnell, N. R. \& Moores, D. L. 1994, Atomic Data Nucl. Data Tabl., 57, 329

Bhatia, A. K., Mason, H. E. \& Blancard, C. 1995, pre-print

Brickhouse, N. S., Edgar, R., Kaastra, J., Kallman, T., Liedahl, D., Masai, K., Monsignori Fossi, B., Petre, R., Sanders, W., Savin, D. W. \& Stern, R. 1995, preprint

Brickhouse, N. S., Raymond, J. C. \& Smith, B. W. 1995, ApJS, in press

Burgess, A. \& Tully, J. A. 1992, A\&A, A\&A, 254, 436

Cornile, M., Dubau, J. A., Mason, H. E., Blancard, C. \& Brown, W. A. 1993, in UV and X-ray Spectroscopy of Laboratory and Astrophysical Plasmas, ed. E. Silver \& S. Kahn, Cambridge: Cambridge Univ., 101

Cornille, M., Dubau, J. A., Mason, H. E. \& Blancard, C. 1995, pre-print

DoscheK, G. A. \& CoWAN, R. D. 1984, ApJS, Ser., 56, 67

Harrison, R. A. \& SaWyer, E. C. 1992, in Proc. of the First SOHO Workshop, ESA SP-348, 17

Jordan, C. 1995, Astron. Soc. of the Pacific, in press

KaAstra, J. J. \& Mewe, R. 1993, Legacy, 3, 16

Kato, T. 1976, ApJS, Ser., 30, 397 
LANG, J. 1994, Atomic Data Nucl. Data Tabl., 57

Landini, M \& Monsignori Fossi, B. C. 1972, A\&AS, Ser., 7, 291

LANDini, M. \& Monsignori Fossi, B. C. 1990, A\&AS, Ser., 82, 229

LANDini, M. \& Monsignori Fossi, B. C. 1991, A\&AS, Ser., 91, 183

Lemaire, P., Wilhelm, K., Axford, W. I., Curdt, W., Gabriel, A. H., Grewing, M., Huber, M. C. E., Jordan, S. D., Kuehne, M., Marsch, E., Poland, A. I., Thomas, R. J., TimothY, G. J., \& VIAL, J. -C. 1992, in Proc. of the First SOHO Workshop, ESA SP-348, p13

Lemen, J. R., Mewe, R., Schrijver, C. J. \& Fludra, A. 1989, ApJ, 341, 474

Masai, K. 1984, Astrophys. and Space Sci., 98, 367

MASAI, K. 1994a, JQSRT, 51, 211

MASAI, K. 1994b, ApJ, 437, 770

Mason, H. E., Bhatia, A. K., Kastner, S. O., Neupert, W. M. \& Swartz, M. Sol. Phys., 92,199

Mason, H. E. \& Monsignori Fossi, B. C. 1994, The A\&A Rev., 6, 123

Mason, H. E. 1994, Atomic Data Nucl. Data Tabl., 57, 305

MEwE, R. 1972, Sol. Phys., 22, 459

Mewe, R. \& Gronenschild, E. H. B. M. 1981, A\&AS, Ser., 45, 11

Mewe, R., Gronenschild, E. H. B. M. \& VAN, DEN, oord, G. H. J. 1985, A\&AS, Ser., 62, 197

Mewe, R. 1990, in Physical Processes in Hot Cosmic Plasmas, 39, ed. W. Brinkmann et al., Dordrecht: Kluwer Acad. Publ.

Monsignori Fossi, B. C. \& Landini, M. 1994, Sol. Phys., 152, 81

Moores, D. L. \& Reed, K. J. 1995, Advan. Atomic. Mol. Phys., in press

Neupert, W. M., Epstein, G. L., Thomas, R. J. \& Thompson, W. T. 1992, Sol. Phys., 137,87

RAYmond, J. C. 1988, in Hot Thin Plasmas in Astrophysics, ed. R. Pallavicini, Dordrecht: Kluwer Acad. Publ., 3

RAYMOND, J. C. \& SMith, B. W. 1977, ApJS, 35, 419

Sampson, D. H. \& Zhang, H. L. 1992, Phys. Rev. A, 45, 1556

SANDERS, W. 1995, these proceedings

SchmitT, J. H. M. M. 1992, in The Seventh Cambridge Workshop on Cool Stars, Stellar Systems, and the Sun, ASP Conf. Ser., 26, 83

SCHRiJVer, C. J., LeMEN, J. R. \& Mewe, R. 1989, ApJ, 341, 484

Storey, P. J., Mason, H. E. \& Saraph, H. E. 1995, preprint

Thomas, R. J. \& Neupert, W. M. 1994, ApJS, 91, 461

VAN, Regemorter, H. 1962, ApJ, 136, 906

Young, P. R., Mason, H. E., Bhatia, A. K., DoscheK, G. A. \& Thomas, R. J. 1995, these proceedings 